\title{
Dielectric analysis of heterogeneous biological tissues based on mixing rule ${ }^{1}$
}

\author{
Liang Zhang ${ }^{\mathrm{a}}$, Peiguo Liu ${ }^{\mathrm{b}}$, Xiuzhen Dong ${ }^{\mathrm{c}}$, Dongming Zhou ${ }^{\mathrm{b},{ }^{*}}$ and Xuetao Shi ${ }^{\mathrm{c}, *}$ \\ ${ }^{a}$ College of Science, National University of Defense Technology, Changsha, Hunan 410073, P.R. \\ China \\ ${ }^{b}$ College of Electronic Science and Engineering, National University of Defense Technology, \\ Changsha, Hunan 410073, P.R. China \\ ${ }^{c}$ Faculty of Biomedical Engineering, Fourth Military Medical University, Xi'an, Shaanxi, 710032, P.R. \\ China
}

\begin{abstract}
Thus far, the measurement of dielectric properties of biological tissues has been achieved on the assumption that the biological tissues are homogeneous. In fact, most tissues should be heterogeneous because there are many small structures included in these tissues, such as blood vessel, nerve fiber and so on. When the dielectric properties of these tissues are measured by conventional sensor, the results are not the dielectric properties of tissues but the effective dielectric properties of the mixture. In this paper, the influence of the inclusion in tissues on the measurement of dielectric properties of heterogeneous biological tissues is studied and the analysis of the effective dielectric properties of heterogeneous tissues based on the mixing rule is proposed. When the coaxial probe is used to measure the dielectric properties of tissue, the results are relative to the dielectric properties of inclusion, dielectric properties of background tissue and the effective volume fraction of inclusion. Therefore, the dielectric properties of inclusion could be calculated according to mixing rule, after the effective dielectric properties are measured and the effective volume fraction of inclusion is estimated.
\end{abstract}

Keywords: Dielectric properties, heterogeneous biological tissues, mixing rule, effective volume fraction

\section{Introduction}

Dielectric properties that are inherent properties of life absorbing and coupling the electromagnetic energy in electromagnetic field are important factors to investigate biological electromagnetism. They are the basal elements to build realistic numerical phantoms of biology for validation or testing in many experiments $[1,2]$. In the radiation therapy, the dielectric properties of tissues are used to analyze and estimate the absorbed energy of normal and abnormal tissues [3]. In diagnosis of disease, the dielectric properties are basic factors of biomedical electrical magnetic function imaging [4]. In

\footnotetext{
${ }^{1}$ Dongming Zhou and Xuetao Shi contributed equally to this work.

* Address for correspondence: Dongming Zhou, College of Electronic Science and Engineering, National University of Defense Technology, Changsha, Hunan 410073, P.R. China. Tel.: (0731)84576219; Fax: 0731-84518730; E-mail: dmzhou@nudt.edu.cn.

Xuetao Shi, Faculty of Biomedical Engineering, Fourth Military Medical University, Xi'an, Shaanxi, 710032, P.R. China. Tel.: (029)84776397; Fax: 029-84776397; E-mail: shixuetao@fmmu.edu.cn.
} 
biological communications, the dielectric properties are also needed to investigate the mechanism of bioelectromagnetic interactions [5].

Dielectric properties are macroscopic properties that describe the physical characteristics of one part. Therefore, the measurements of dielectric properties of biology are usually achieved on tissues [6]. From the interim of last century, a considerable amount of dielectric properties data has been measured [7-9]. However, these measurements are achieved on the assumption that the biological tissues are homogeneous. In fact, most tissues should be heterogeneous because there are many small structures or matters included in these tissues such as blood vessel, nerve fiber even some other differential tissues. The researcher considered the difference between the whole tissue and isolated inclusion in some measurements of tissue such as blood [10]. But there is still a continuing need for the measurement of other biological tissues.

On the other hand, the biomedical electrical magnetic imaging could achieve measuring the dielectric properties of different inclusions in the tissue. But this method needs the prior information to improve the resolution. It means when the biomedical electrical magnetic imaging is used in measurement, this method needs plenty of information about the inclusions in tissue, such as the volume, position and estimated dielectric properties of inclusions for improving the precision of measurement. The volume and position of inclusions could be derived by CT or MRI [2]. But it is hard to estimate dielectric properties value of inclusions. Generally, it is first to determine what the inclusions are. Then the dielectric data of the inclusions in literature is used as the estimated dielectric properties. But to different individuals, the dielectric properties of the same inclusion may be different. It is not suitable to use the literature data as the estimated dielectric properties.

This study simulated and analyzed the influence of the inclusion in tissues on the dielectric measurement of heterogeneous biological tissues. The relationship between the effective dielectric properties of heterogeneous tissues measured by coaxial probe and the dielectric properties of inclusion was proposed based on the mixing rule. According to this relationship, the dielectric properties of inclusion could be calculated, after the effective dielectric properties were measured and the effective volume fraction of inclusion was estimated.

\section{Dielectric measurement using coaxial probe and mixing rule}

The open-ended coaxial probe is usually used to measure the dielectric properties of biological tissues [11-13]. When the probe is terminated by tissues, the terminal input admittance of probe $Y_{\text {in }}$ that contains the dielectric information of tissues could be measured. Finally, the dielectric properties could be calculated according to follow expression [14]:

$$
Y_{i n}=j \omega C_{f}\left(\varepsilon_{r}^{\prime}\right)+j \omega C_{0} \varepsilon_{r}+G\left(\varepsilon_{r}^{\prime}, \sigma, \omega\right)+j b_{0}
$$

When the tissue that contains other differential inclusion is heterogeneous, the results derived by coaxial probe are not the dielectric properties of test tissue but the effective dielectric properties of whole tissue with the inclusion. Because the input admittance $Y_{\text {in }}$ measured by probe describes the whole measurement area of tissue, It is hard to distinguish the test tissue and inclusion. This problem is also found in other measurement method such as impedance measurement. Therefore, it needs some other method to distinguish the dielectric properties of test tissue and included tissue.

Sihvola discussed the effective dielectric properties of heterogeneous materials, in particular the 
media with lossy constituents that have complex permittivity parameters [15]. Several mixing rules were presented by which the effective dielectric property parameters of the mixture could be calculated as a function. Maxwell Garnett mixing rule was basic expression to calculate the effective dielectric properties of the simple mixture where the spherical inclusions were included in a homogeneous background medium:

$$
\varepsilon_{e f f}=\varepsilon_{e}+3 v \varepsilon_{e} \frac{\varepsilon_{i}-\varepsilon_{e}}{\varepsilon_{i}+2 \varepsilon_{e}-v\left(\varepsilon_{i}-\varepsilon_{e}\right)}
$$

Where $\varepsilon_{\text {eff }}$ is the effective complex permittivity of mixture, $\varepsilon_{\mathrm{e}}$ is the complex permittivity of background medium, $\varepsilon_{\mathrm{i}}$ is the complex permittivity of inclusions, and $v$ is the volume fraction of inclusions in the mixture. Eq. (2) was based on the assumption that the spherical inclusions and the electromagnetic field both were evenly distributed in the background medium. In fact, it was hard to be achieved in the measurement of tissues.

However, the shape and size of inclusions could be ignored when the work wavelength was much larger than the size of inclusions. On the other hand, the detecting area of open-ended coaxial probe was not large, and the electromagnetic field in the tissues could be approximate considered to be evenly distributed. As a result, when the open-ended coaxial probe was used to measure the dielectric properties of heterogeneous tissues in low frequency, Maxwell Garnett mixing rule may also be utilized to describe the relationship between the effective dielectric properties of heterogeneous tissues and the dielectric properties of included tissues.

\section{Data generation and simulation settings}

The dielectric measurement of heterogeneous skin tissue which included the blood was simulated to validate whether the Maxwell Garnett mixing rule was suitable to the open-ended coaxial probe measurement of dielectric properties of heterogeneous tissues.

\subsection{Simplified model of heterogeneous skin tissue}

In the model of heterogeneous skin tissue, the blood was taken as the inclusion in the skin tissue which was similar to the symptom of subcutaneous hemorrhage. The dielectric properties of skin and blood that were important parameters for the simulation model could be calculated by Gabriel's fourpole Debye model [8]:

$$
\varepsilon_{r}^{*}=\varepsilon_{\infty}+\sum_{i=1}^{4} \frac{\Delta \varepsilon_{i}}{1+j \omega \tau_{i}}+\frac{\sigma_{s}}{j \omega \varepsilon_{0}}
$$

In Eq. (3), $\varepsilon_{\infty}$ is the high frequency permittivity and $\sigma_{\mathrm{s}}$ is the static conductivity. $\tau$ is the relaxation time, and $\alpha$ is the relaxation factor. These parameters of skin and blood were given in [8].

\subsection{Simulation settings}

Figure 1 shows the diagram of simulation settings. Skin was considered to be the background tissue 


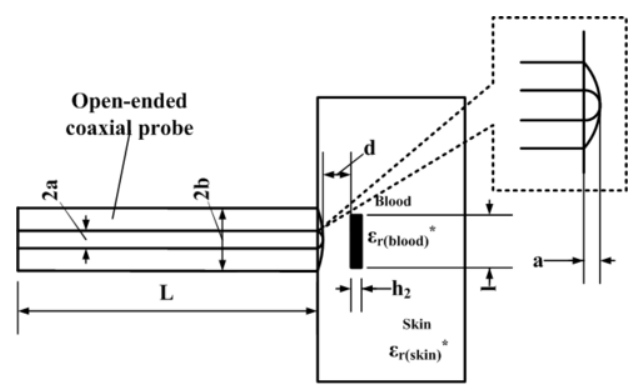

(a)

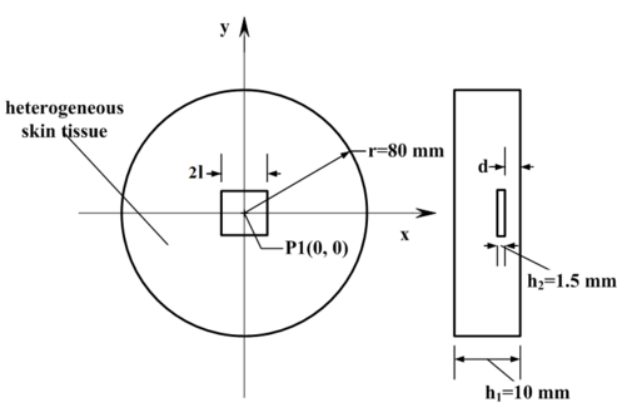

Fig. 1. The diagram of simulation settings: (a) the open-ended coaxial probe and test tissue (b) the heterogeneous skin tissue.
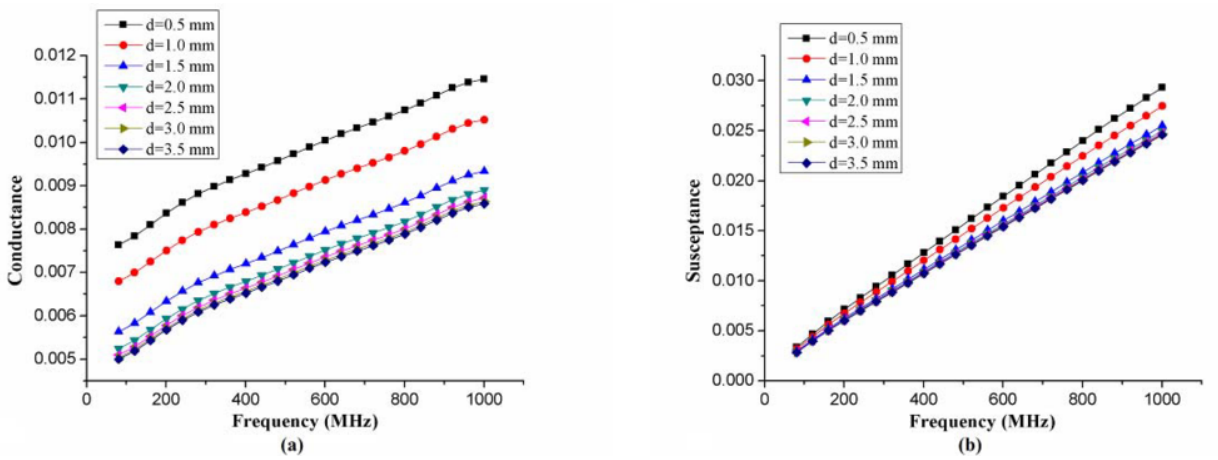

Fig. 2. The changes of input admittance $Y_{\text {in }}$ with d: (a) the real component of $Y_{\text {in }}(b)$ the imaginary component of $Y_{\text {in }}$.

which was set up as a single cylinder with a radius $r=80 \mathrm{~mm}$ and a thickness $h_{1}=10 \mathrm{~mm}$. Blood were taken as the inclusion which was set up as a cube with the thickness $\mathrm{h}_{2}=1.5 \mathrm{~mm}$ and the sectional length 21. The depth of the blood in the skin was set to be $d$. The dimensions of coaxial sensor in the simulation were set as follows: The opening radius of probe was $b=4 \mathrm{~mm}$ and inner radius was $\mathrm{a}=1.303 \mathrm{~mm}$. The space between the inner conductor and outer conductor was filled with air. The inner conductor was set to be $\mathrm{a}=1.303 \mathrm{~mm}$ longer than outer conductor. The length of the probe $\mathrm{L}$ was set up to be $30 \mathrm{~mm}$. The working frequency of detection was set to be from $80 \mathrm{MHz}$ to $1 \mathrm{GHz}$.

\section{Results and discussion}

\subsection{The effective detecting area of open-ended coaxial probe}

The detecting area of open-ended coaxial probe is not large. Therefore, it is necessary to determine the effective detecting area of the probe when the probe is terminated by skin. Figure 2 shows the changes of input admittance $Y_{\text {in }}$ with the depth of the blood in the skin. The sectional length of blood was set to be $1=15 \mathrm{~mm}$.

As shown in Figure 2, the input admittance $Y_{\text {in }}$ did not changed with the depth of the blood after $d$ was larger than $3.0 \mathrm{~mm}$. It means that it is hard for the probe to detect the blood. The detecting depth of probe is approximate to $3.0 \mathrm{~mm}$. On the other hand, the detecting area of probe on the different depth was also investigated. Figures 3 and 4 show the changes of input admittance $Y_{\text {in }}$ with the sectional length of the blood on different values of depth in the skin. It was shown in Figures 3 and 4 


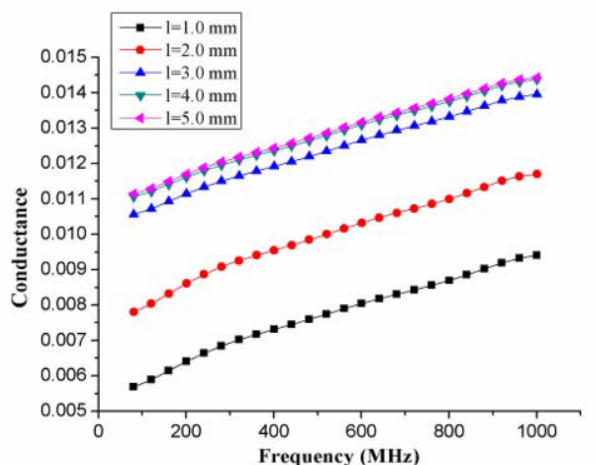

(a)

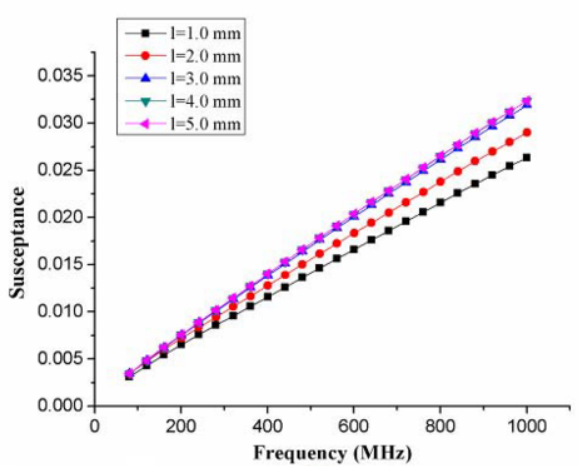

(b)

Fig. 3. The changes of input admittance $Y_{\text {in }}$ with $1(d=0 \mathrm{~mm})$ : (a) the real component of $Y_{\text {in }}(b)$ the imaginary component of $\mathrm{Y}_{\text {in }}$.
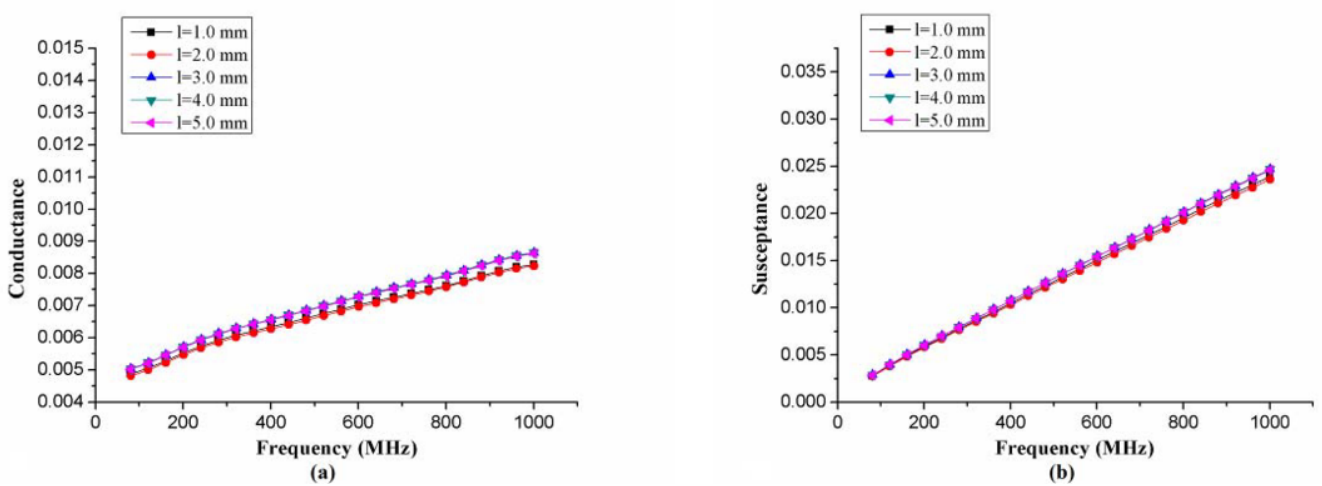

Fig. 4. The changes of input admittance $Y_{\text {in }}$ with $1(d=3.0 \mathrm{~mm})$ : (a) the real component of $Y_{\text {in }}(b)$ the imaginary component of $\mathrm{Y}_{\mathrm{in}}$.

that the detecting area of probe was the largest when the depth of blood was $0 \mathrm{~mm}$, and the detecting area of probe was almost one point when the depth of blood was $3.0 \mathrm{~mm}$. Therefore, the detecting area of probe could be described as a cone with the height $\mathrm{h}=3.0 \mathrm{~mm}$ and the radius of underside $\mathrm{r}=4.0 \mathrm{~mm}$.

\subsection{The effective volume fraction of blood and Maxwell Garnett mixing rule}

According to the result of prior section, the volume fraction $v$ in Eq. (2) should not be considered as the proportion of blood in the skin, but the proportion of blood in the detecting area of probe, as shown in Figure 5. As a consequence, the volume fraction $v$ in Eq. (2) was the effective volume fraction of blood in the detecting area when the coaxial probe was used to measure the dielectric properties of heterogeneous tissues.

In order to validate the applicability of the Maxwell Garnett mixing rule in dielectric measurement of heterogeneous tissues, the effective dielectric properties $\varepsilon_{\text {eff }}$ of heterogeneous skin tissues with the different values of depth of blood in Figure 2 were calculated according to the Maxwell Garnett mixing rule. Figure 6 shows the effective dielectric results of measurement by Eq. (1) and the results of calculation by Eq. (2). The volume fraction $\mathrm{v}$ of blood on the different values of depth was given in Table 1.

As shown in Figure 6, the values of effective dielectric properties fell in between skin and blood. 


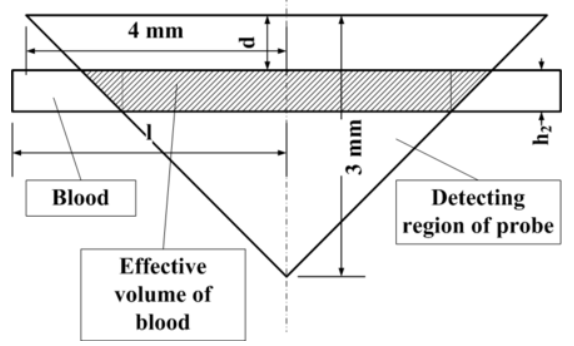

Fig. 5. The effective volume of blood in the detecting area of probe.
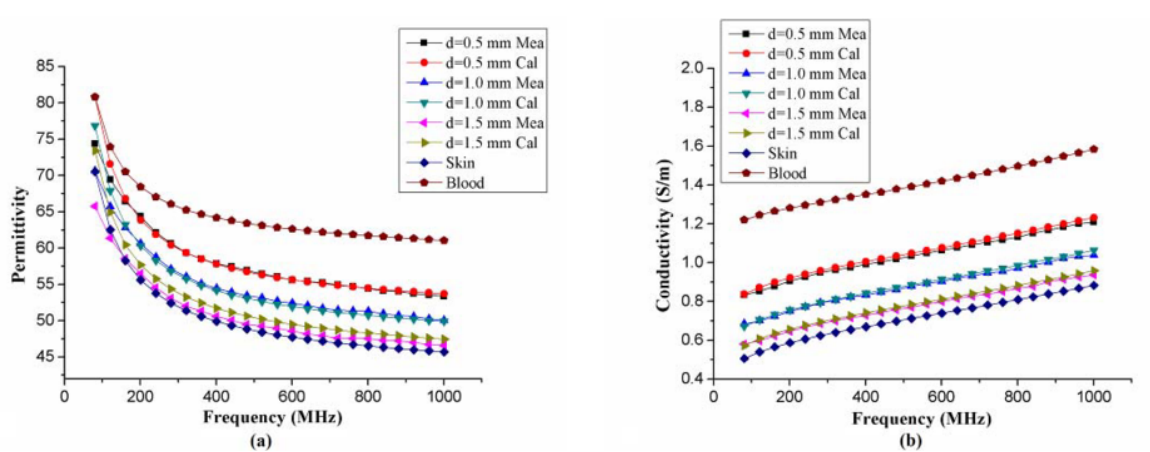

Fig. 6. The effective dielectric properties of heterogeneous skin tissues with the different depth of blood: (a) Permittivity (b) Conductivity.

Table 1

The volume fraction $\mathrm{v}$ of blood on the different depth

\begin{tabular}{l|l|l|l}
\hline The volume fraction & $\mathrm{d}=0.5(\mathrm{~mm})$ & $\mathrm{d}=1.0(\mathrm{~mm})$ & $\mathrm{d}=1.5(\mathrm{~mm})$ \\
\hline $\mathrm{v}$ & $54.2 \%$ & $29.2 \%$ & $12.5 \%$ \\
\hline
\end{tabular}

According to Eq. (2), it satisfied the limiting processes for vanishing blood and skin phases:

$$
\begin{aligned}
& v \rightarrow 0 \quad \Rightarrow \quad \varepsilon_{\text {eff }} \rightarrow \varepsilon_{e} \\
& v \rightarrow 1 \quad \Rightarrow \quad \varepsilon_{\text {eff }} \rightarrow \varepsilon_{i}
\end{aligned}
$$

On the other hand, the effective dielectric results of measurement by Eq. (1) and the results of calculation by Eq. (2) agreed with each other very well according to Figure 6. This means the Maxwell Garnett mixing rule could be used to analyze the dielectric measurement of heterogeneous tissues. The errors of analysis based on mixing rule resulted from the estimation of the volume fraction of inclusion.

\section{Conclusion}

In this paper, Maxwell Garnett mixing rule was proposed to analyze the dielectric measurement of heterogeneous tissues using the open-ended coaxial probe. The influence of the inclusion in tissues on the measurement of dielectric properties of heterogeneous biological tissues was simulated and analyzed based on the model of heterogeneous skin tissue. According to the results of simulation, the detecting area of open-ended coaxial probe was not large that could be approximate described as a 
cone area. The Maxwell Garnett mixing rule could be used to analyze the dielectric measurement of heterogeneous tissues. However, the volume fraction $\mathrm{v}$ was not the proportion of inclusion in the background tissue, but the effective volume fraction of inclusion in the detecting area. When the volume fraction was estimated, the dielectric properties of inclusion in heterogeneous tissue could be calculated according to the measured effective dielectric properties based on Maxwell Garnett mixing rule. In future work, the actual measurement would be performed for further verification. The depth of detection and resolution of the probe should be improved for more sophisticated type of inclusions.

\section{Acknowledgments}

This study was partially supported by the National Science and Technology Pillar Program of China under grant 2012BAI19B01, the National Natural Science Foundation of China under grant 61372029 and the Scientific Research Innovation Project for Graduate Student under grant CX2013B011.

\section{References}

[1] J. Garrett and E. Fear, Stable and flexible materials to mimic the dielectric properties of human soft tissues, IEEE Antennas and Wireless Propagation Letters 13 (2014), 599-602.

[2] J.D. Shea, P. Kosmas, B.D. Van Veen, et al., Contrast-enhanced microwave imaging of breast tumors: A computational study using 3D realistic numerical phantoms, Inverse Problem 26 (2010), 074009.1-074009.22.

[3] M-Reza N-Zadeh, C.H. Chapman, T.S. Lawrence, et al., Uncertainty in assessment of radiation-induced diffusion index changes in individual patients, Physics in Medicine and Biology 58 (2013), 4277-4296.

[4] S.Y. Semenov, A.E. Bulyshev, A. Abubakar, et al., Microwave-tomographic imaging of the high dielectric-contrast objects using different image-reconstruction approaches, IEEE Trans on Microwave Theory and Techniques 53 (2005), 2284-2294.

[5] C. Rossi, A. Foletti, A. Magnani, et al., New perspectives in cell communication: Bioelectromagnetic interactions, Seminars in Cancer Biology 21 (2011), 207-214.

[6] P. Wang and C.L. Brace, Tissue dielectric measurement using an interstitial dipole antenna, IEEE Trans on Biomedical Engineering 59 (2012), 115-121.

[7] C. Gabriel, S. Gabriel and E. Corthout, The dielectric properties of biological tissues: I. Literature survey, Physics in Medicine and Biology 41 (1996), 2231-2249.

[8] S. Gabriel, R. Lau and C. Gabriel, The dielectric properties of biological tissues: III. Parametric models for the dielectric spectrum of tissues, Physics in Medicine and Biology 41 (1996), 2271-2293.

[9] A. Peyman and C. Gabriel, Dielectric properties of rat embryo and foetus as a function of gestation, Physics in Medicine and Biology 57 (2012), 2103-2116.

[10]N.S. Selim, Comparative study on the effect of radiation on whole blood and isolated red blood cells, Romanian Journal of Biophysics 20 (2010), 127-136.

[11] C. Gabriel, T.Y.A. Chan and E.H. Grant, Admittance models for open ended coaxial probes and their place in dielectric spectroscopy, Physics in Medicine and Biology 39 (1994), 2183-2200.

[12]D. Popovic, L. McCartney, C. Beasley, et al., Precision open-ended coaxial probes for in vivo and ex vivo dielectric spectroscopy of biological tissues at microwave frequencies, IEEE Trans on Microwave Theory and Techniques $\mathbf{5 3}$ (2005), 1713-1722.

[13]E. Piuzzi, C. Merla, G. Cannazza, et al., A comparative analysis between customized and commercial systems for complex permittivity measurements on liquid samples at microwave frequencies, IEEE Trans on Instrumentation and Measurement 62 (2013), 1034-1046.

[14] L. Zhang, X.T. Shi, F.S. You, et al., Improved circuit model of open-ended coaxial probe for measurement of the biological tissue dielectric properties between megahertz and gigahertz, Physiological Measurement 34 (2013), N83N96.

[15]A. Sihvola, Mixing rules with complex dielectric coefficients, Subsurface Sensing Technologies and Applications 1 (2000), 393-415. 